(RESEARCH ARTICLE)

\title{
ROR1 expression in chronic lymphocytic leukemia and the effect of CD5
}

\author{
Hessah Saeed Alsulami 1, 2, ${ }^{*}$, Nora Saeed Ali ${ }^{2}$, and Jimi Maro ${ }^{2}$ \\ ${ }^{1}$ Prince Mohammed hospital, Lab. \& blood bank, Riyadh, Saudi Arabia, Arabian Gulf university, Manamah, Bahrain. \\ 2 Saudi ministry of health, Riyadh, Saudi Arabia
}

Publication history: Received on 13 July 2020; revised on 17 August 2020; accepted on 20 August 2020

Article DOI: https://doi.org/10.30574/wjarr.2020.7.3.0260

\begin{abstract}
B cell chronic lymphocytic leukemia (CLL) is characterized by accumulation of monoclonal CD5+ mature B cells. The expression of CD5 plays a role in the malignant behavior of CLL cells via controlling the expression of some genes that enhance the expression of; apoptosis inhibitors BCL-2, NF- $\mathrm{B}$, Wnt, and cytokine. By being naturally phosphorylated on tyrosines, CD5 is chronically activated in CLL cells. Moreover, B CLL cell also characterized by the expression of ROR1. In this study we wanted to know if CD5 has an effect on the surface expression of ROR1 in CLL cells via comparing the mean fluorescence intensity (MFI) between CD5 and ROR1.Therefore, A cross-sectional study of immune-phenotype results for 50 randomly selected patients diagnosed with CLL in the period from June 2014 till Jan.2016 was performed. 28 cases were excluded because ROR1 test was not performed for them. The study was carried out on 22 patients. The correlation between CD5 MFI and ROR1 MFI was assessed using Spearman's correlation. We found that in 21 out of 22 cases the correlation was significant with $\mathrm{P}<0.001$ and correlation coefficient ( $\mathrm{rs}$ ) of 0.788 . This study gives us a hint that CD5 might have an effect on the expression of ROR1 in CLL lymphocytes. This finding needs to be supported by experimental study looking at the mechanism by which CD5 has such an effect.
\end{abstract}

Keywords: ROR1; CD5; CLL; MFI

\section{Introduction}

B cell chronic lymphocytic leukemia (CLL) is characterized by accumulation of monoclonal CD5+ mature B cells [1]. CD5 is known to be a T lymphocytes marker, but it is weakly expressed on a small subgroup of B lymphocytes [2]. The expression of CD5 plays a role in the malignant behavior of CLL cells via regulating the expression of some genes that enhance the expression of apoptosis inhibitors BCL-2, NF- $\kappa \mathrm{B}$, Wnt, and some cytokines [3]. By being naturally phosphorylated on tyrosines, CD5 is chronically activated in CLL cells [4], which might be the cause for constitutive phosphorylation of the transcriptional factor STAT-3.

B CLL cell also characterized by the expression of Receptor tyrosine kinase-like orphan receptor 1 (ROR1) [5]. Expression of ROR1 ordinarily is confined to early embryogenesis, where it contributes to organogenesis [6-8]. However, ROR1 is not detected on postpartum tissues, including hematopoietic stem cells, except on a small subset of B-cell precursors, called hematogones [9]. The expression of ROR1 in CLL raise the question of whether it has a role in the pathogenesis of the disease or not. Knowing that the number of ROR1 receptors on the surface of CLL cells was estimated to be in the range of 10,000/cells [10] and its expression is increased with the progression of the disease [11] support its role in the pathogenesis of CLL. Also, it has been shown that STAT3 may be involved as a promoting factor in the expression of ROR1 [12].

In this study we tested the hypothesis that CD5, by being constitutive phosphorylated, affects the expression of ROR1 via activation of STAT-3 (figure-1). To do so, a cross- sectional study was conducted to compare MFI between CD5 and ROR1 in CLL samples.

\footnotetext{
* Corresponding author: H. S. Alsulami
} 


\section{Material and methods}

\subsection{Patient samples}

The diagnostic immune-phenotype results for 50 randomly selected patients diagnosed with CLL in the period from June 2014 till Jan. 2016 were reviewed, after getting the approval of the ethics committee at the university. 28 cases were excluded because ROR1 test was not performed for them. The study was carried out on 22 patients.

\subsection{Flow cytometry}

The immunophenotyping was carried out using BD FACSCanto II (Becton Dickinson) and CLL panel which composed of CD45, CD19, CD5, CD20, CD23, CD79b, CD38, Kappa, Lambda antibodies (Becton Dickinson) in addition to affinitypurified polyclonal goat anti-human ROR1 (R\&D Systems). The data was analyzed using FACSDiva software (Becton Dickinson). The expression of R0R1 was assessed for CD19, CD5 positive cells.

\subsection{Statistical analysis}

Statistical analysis was performed with SPSS (IBM SPSS Statistics Version 26) using Spearman's correlation.

\section{Results}

Cells from 21 out of 22 cases (96\%) showed typical CLL immunophenotype which was moderate to strong expression for CD19, CD5, and CD23 and dim for CD20 and CD79b. 12\% of the cases were monoclonal for Kappa, while the rest were for lambda. Half of the cases in this study moderately expressed CLL prognostic marker CD38. All cases expressed ROR1 but the strength, (MFI), ranged from strong to dim.

In those 21 cases there was some relation between the surface expression of CD5 and ROR1, whenever the expression of CD5 was dim the expression of ROR1 was also dim and vis versa. To assess the significance of this relation we run Spearman's correlation test and found that (figure-2) only in one case this correlation did not exist, this case showed atypical CLL immunophenotype with weakly expression of CD23 and ROR1 and strongly expressed CD5 and CD20.

\section{Discussion}

CLL is a B lymphocyte malignancy that characterizes by CD5 expression. It is diagnosed when there is an accumulation of malignant lymphocytes in peripheral blood, bone marrow and lymph-nodes [13]. The search for the pathogenesis of this malignancy focused on the role of B-cell receptor (BCR), CD5 and other proteins [14]. In this study we tried in an observational way to look at the effect that CD5 might have on the expression of ROR1 and in turn the malignant nature of the disease.

CD5 marker in B lymphocytes is located in a physical binding with BCR and acts as a negative regulator of down-stream signaling pathways [15]. In addition, via activation of STAT-3 and nuclear factor of activated T cells (NFAT), CD5 is directly played a role in the production of anti-apoptotic proteins such as IL-10 [3-4]. The production of IL-10 is significantly associated with CLL progression because it acts as a growth factor for CLL cells by increasing proliferation and protection against apoptosis $[4,16,17]$.

STAT3 is a cytoplasmic transcription factor that upon phosphorylation moves to the nucleus, binds to DNA, and activates STAT3-regulated genes [4]. CLL cells are characterized by STAT3 being constitutively phosphorylated on serine 727 residues which is in turn activates antiapoptotic genes [18]. This constitutive activation could be as a result of CD5 being constitutively active and due to that STAT3 has the ability to activates ROR1 gene which leads to the expression of ROR1 in CLL cells [12]. During embryonic developmental stage, STAT3 is a very critical protein and its absence leads to embryonic lethality as well as ROR1 [19]. Also, in embryonic stage STAT3 does not need to be activated by other proteins to act [20] which means it might be constitutively active and it is responsible for the expression of ROR1 by embryonic tissue. 


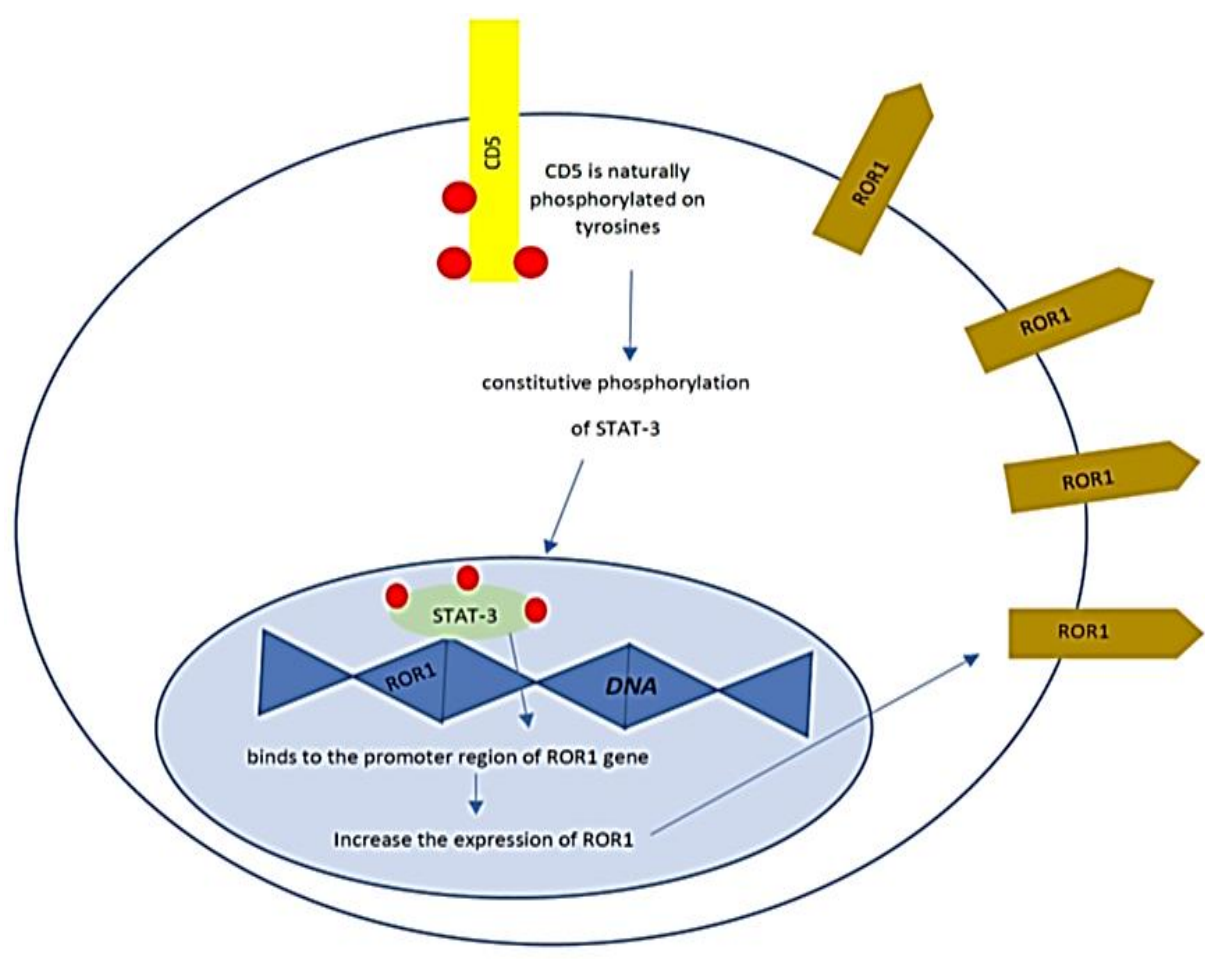

= Phosphate.

Figure 1 The effect of CD5 on the expression of ROR1 (Study hypothesis)

Depending in the above-mentioned facts, we in this study tried to find an indirect evidence for the effect of CD5 via STAT3 on the expression of ROR1 by CLL cells by comparing MFI between anti-CD5 and Anti-ROR1. MFI is directly proportion to the amount of the antigen on the surface of CLL lymphocytes, which means the higher MFI for a given antigen the higher the numbers of that antigen on the cell surface. Therefore, when we compared MFI for CD5 in the study samples with that for ROR1 we observed that whenever MFI for CD5 is high The MFI for ROR1 is high too and vis versa. This correlation between the two markers proved to be significant.

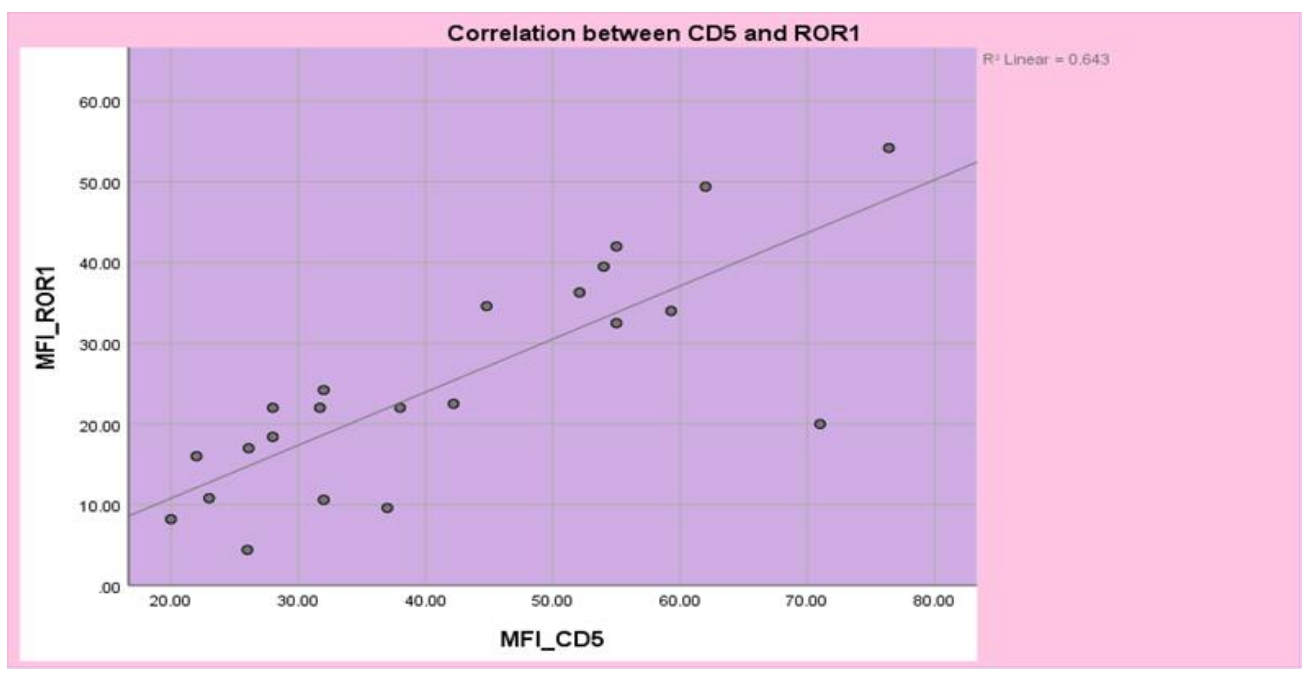

Figure-2 the correlation graph between CD5 MFI (independent variable) and ROR1 MFI (dependent variable) 


\section{Conclusion}

The result of this study raises some questions such as; Dose CD5 being constitutively active play a role in the pathogenesis of CLL? Is there any relation between CD5 and STAT3? Dose CD5 affect the expression of R0R1? Is this effect of CD5 on the expression of ROR1 explain why mantle cell lymphoma (MCL), a CD5 positive B-cell malignancy, also express ROR1? To answer all of these questions another study should be carried out to investigate the cause for CD5 being constitutively active in CLL lymphocytes and its role in STAT3 being also constitutively active in that cells as well as the role of both on the expression of ROR1.

\section{Compliance with ethical standards}

\section{Acknowledgments}

The author would like to thank all lab. Staff at university hospital.

\section{Disclosure of conflict of interest}

The author declares no conflict of interest.

\section{Statement of informed consent}

Informed consent was obtained from all individual participants included in the study.

\section{References}

[1] Thakral B, Medeiros LJ, Desai P, et al. (2017). Prognostic impact of CD5 expression in diffuse large B-cell lymphoma in patients treated with rituximab-EPOCH. Eur J Haematol, 98, 41521.

[2] Dalloul A. (2009). CD5: a safeguard against autoimmunity and a shield for cancer cells. Autoimmun Rev, 8, 349353.

[3] Mageed RA, Garaud S, Taher TE, et al. (2012). CD5 expression promotes multiple intracellular signaling pathways in B lymphocyte. Autoimmun Rev, 11, 795-798.

[4] Garaud S, Morva A, Lemoine S, et al. (2011). CD5 promotes IL-10 production in chronic lymphocytic leukemia B cells through STAT3 and NFAT2 activation. J Immunol, 186, 4835-4844.

[5] Baskar S, Kwong KY, Hofer T, et al. (2008). Unique cell surface expression of receptor tyrosine kinase ROR1 in human B-cell chronic lymphocytic leukemia. Clin Cancer Res, 14, 396-404.

[6] Matsuda T. (2001). Expression of the receptor tyrosine kinase genes, Ror1 and Ror2, during mouse development. Mech Dev, 105(1-2), 153-156.

[7] Al-Shawi R, Ashton SV, Underwood C and Simons JP. (2001). Expression of the Ror1 and Ror2 receptor tyrosine kinase genes during mouse development. Dev Genes Evol, 211(4), 161-171.

[8] Lyashenko N. (2010). Mice lacking the orphan receptor ror1 have distinct skeletal abnormalities and are growth retarded. Dev Dyn, 239(8), 2266-2277.

[9] Broome HE, Rassenti LZ, Wang HY, et al. (2011). ROR1 is expressed on hematogones (non-neoplastic human Blymphocyte precursors) and a minority of precursor-B acute lymphoblastic leukemia. Leuk Res, 35(10), 13901394.

[10] Hojjat-Farsangi M, Khan AS, Daneshmanesh AH, et al. (2013). The tyrosine kinase receptor ROR1 is constitutively phosphorylated in chronic lymphocytic leukemia (CLL) cells. PLoS One, 7, 83-93.

[11] Zhang Z and Zhang P. (2016). Receptor tyrosine kinases in carcinogenesis. Oncology Letters, 12, 3679-3682.

[12] Borcherding N, Kusner D, Liu GH, et al. (2014). ROR1, an embryonic protein with an emerging role in cancer biology. Protein Cell, 5, 496-502.

[13] Chiorazzi N, Ferrarini M, et al. (2005). Chronic lymphocytic leukemia. N. Engl. J. Med, 352, 804-815.

[14] Alhakeem SS, Sindhava VJ, McKenna MK, et al. (2015). Role of B cell receptor signaling in IL-10 production by normal and malignant B-1 cells, 1362, 239-49. 
[15] Zhang C, Xin H, Zhang W, et al. (2016). CD5 binds to interleukin-6 and induces a feed-forward loop with the transcription factor STAT3 in B cells to promote cancer. Immun, 44, 913-23.

[16] Gary-Gouy H, Sainz-Perez A, Marteau J-B, et al. (2007). Natural phosphorylation of CD5 in chronic lymphocytic leukemia B cells and analysis of CD5-regulated genes in a B cell line suggest a role for CD5 in malignant phenotype. J Immunol, 179, 4335-44.

[17] Laurenti L, Vannata B, Innocenti I, et al. (2013). The use of monoclonal antibodies in the treatment of autoimmune complications of chronic lymphocytic leukemia. Med J Hematol Infect Dis, 5, 1-10.

[18] Frank D, Mahajan S, Ritz J. (1997). B lymphocytes from patients with chronic lymphocytic leukemia contain signal transducer and activator of transcription (STAT) 1 and STAT3 constitutively phosphorylated on serine residues. J. Clin. Investig, 100, 3140-3148.

[19] Raz R, Lee C, Cannizzaro L, et al. (1999). Essential role of STAT3 for embryonic stem cell pluripotency. Proc Natl Acad Sci, 96, 2846-2851.

[20] Takeda K. (1997). Targeted disruption of the mouse Stat3 gene leads to early embryonic lethality. Proc Natl Acad Sci, 94, 3801-3804. 